\title{
Comparative effect of electroacupuncture with different frequency on headache attacks in migraine outpatients: study protocol for a randomized controlled trial
}

\author{
$\mathrm{Na} \mathrm{Nie}^{1 \#}$, Le Chen ${ }^{2 \#}$, Tong $\mathrm{Li}^{2}$, Chuanlong Zhou ${ }^{1}$, Bangwei $\mathrm{Li}^{1}$, Conghua $\mathrm{Ji}^{3}$, Jie Zhou ${ }^{1}$, Qin \\ Chen ${ }^{1}$, Qiushuang $\mathrm{Li}^{3}$, Yi Liang ${ }^{1,2^{*}}$, Jianqiao Fang ${ }^{1,2}$
}

1Department of Acupuncture, The Third Affiliated Hospital of Zhejiang Chinese Medical University, No. 219 Moganshan Road, Xihu District, Hangzhou, Zhejiang Province 310005, China 2 The Third Clinical Medical College of Zhejiang Chinese Medical University, No. 548 Binwen Road, Binjiang District, Hangzhou, Zhejiang Province 310053, China

3 The Clinical Research Institute of Zhejiang Provincial Hospital of TCM, No.54 Youdian Road, Xihu District, Hangzhou, Zhejiang Province 310006, China.

Full list of author information is available at the end of the article.

* Corresponding author: liangyiwww@126.com

\# contributed equally.

\section{Abstract}

Background: Headache attacks severely impaired life quality and increase economic burden of migraineurs. Electroacupuncture (EA) has been used world-widely to treat several pain-related diseases including migraine. However, whether EA with low- or high-frequency exerts distinct analgesic effect remains unknown and need to further study.

Methods/Design:This study is a randomized, prospective randomized trial with three parallel arms.A total of 129 migraine outpatients will be randomly allocated to $2 \mathrm{~Hz}$ EA group, $100 \mathrm{~Hz}$ EA group and control group.Twelve treatment sessions will be performed over a four-week period. The primary outcome will be measured by frequency of migraine attacksat baseline, four-week of treatment and consecutive four-week follow-up. Secondary outcome measures will benumber of days with migraine, frequency of taking ibuprofen, Visual Analogue Scale (VAS), Self-Rating Anxiety Scale (SAS), Self-Rating Depression Scale (SDS) and Migraine Specific Quality of Life questionnaire (MSQ), safety and compliance evaluation. Discussion:This trial is expected to comparative effect of electroacupuncture with different frequency on headache attacks in migraineur.

Trial registration:Chinese Clinical Trial Registry, ChiCTR-1800017259. Registered on 20 July 2018

Keywords: Study protocol, EA, migraine, Randomized controlled trial

\section{Background}

Migraine is a common and frequentdisorder in department of neurology[1, 2]. It occurs on 
one side or both sides onset of pulsatile headache which can be accompanied by prodromal symptoms such as nausea, vomiting, photophobia, phobia, depression and burnout. Migraine has a long duration, frequent recurrence,difficulty in healing, and even causes severe disability. The World Health Organization (WHO) Report listed migraine was the 19th major cause of disability for many years and the disability caused by severe migraine was regarded as quadriplegia[3-5]. An epidemic survey investigated in the mainland of China showed the yearly prevalence of migraine was 9.3\%(male:5.9\%; female:12.8\%) which was close to the global average of 11\%[6].Epidemiological studies in 2016 demonstrated that global age-standardized prevalence migraine was $14.4 \%$ in all which was $18.9 \%$ for women and $9.8 \%$ for men[7]. More than half of migraineurs reported the quality of daily work and life was affected[1, 2, 8]. Migraineurs suffered from substantial decline in productive capacity,which led to increased economic burden and low-quality of life[9]. It had been reported that total annual direct costs for migraine in China were CNY 58.0 billion (USD 8.4 billion) and the total indirect annual financial losses to society due to migraine-induced lost productivity was CNY 273.7 billion (USD 39.4 billion)[6]. Migraine became the third cause of disability in people under 50 years of age according to the Global Burden of Disease Study 2015 (GBD 2015)[10]. Migraine not only impaired human health, but also aggravated health-related economic burden which further imposed negative impact on quality of life[6, 9, 11, 12]. Thus, how to effectively prevent and control migraine attacks has been a hot area of research, which attract increasing attention of clinical researchers all over the word. Up to now, the mechanisms of migraine attack are not fully understood and lack of migraine-targeted drugs still bother migraineurs. Drugs such as beta-adrenoceptor blockers and antidepressants usually prescribed to alleviate headache attack. However, medication-induced side effects limited clinical effectiveness and reduced patient-based compliance [11, 13].

Electroacupuncture (EA) is one of the most extensively applicated acupuncture therapy and has been used world-widely several decades in treating chronic pain. Accumulating evidences had been demonstrated that EA has good analgesic effect for a variety of pain, including inflammatory pain and neuropathy pain, etc[14-16]. It was general known that the analgesic effect of $2 \mathrm{~Hz}$ EA is superior to $100 \mathrm{~Hz}$ EA for neuropathic pain $[17,18]$. Cochrane systematic reviews indicated that acupuncture or EA has definite curative effect and the advantage for safety on treating migraine[19-21]. Zhao et al further demonstrated true acupuncture has long-term reduction in migraine recurrence long-term effect of acupuncture for migraine [22]. However, therapeutic difference between low-frequency EA and high-frequency EA on migraine remains unknown. Therefore, the study is designed to compare analgesic effect of EA with different frequency, further provide more evidence to select the optimal frequency of EA on migraine.

\section{Methods/Design}

\section{Study design}

Thestudy is a randomized, controlled clinical trial with three parallel arms. It aims to clarify the therapeutic effect of EA with low or high frequency on headache attacks in migraineurs and to screen out the dominant frequency of EA. The trial will be conducted after obtaining consent of the Ethics Committee. Participants will be randomized to the $2 \mathrm{~Hz}$ EA group,the $100 \mathrm{~Hz}$ EA group, and the control group(1:1:1 ratio). The EA group will receive 12 sessions EA therapy in total for 4 weeks and the control group will receive control acupuncture treatment to alleviate pain. The outcome will be assessed after 4 weeks of treatment. Post-treatment 
follow-up will be performed at 4, 8, and 12 weeks after treatment (Figure 1). In all groups, participants will not take any regular medications for migraine, but only allows to take ibuprofen capsules in necessary which must be recorded the frequency. The trial was obtained the registration number ChiCTR-1800017259 in Chinese Clinical Trial Registry (www.chictr.org.cn).

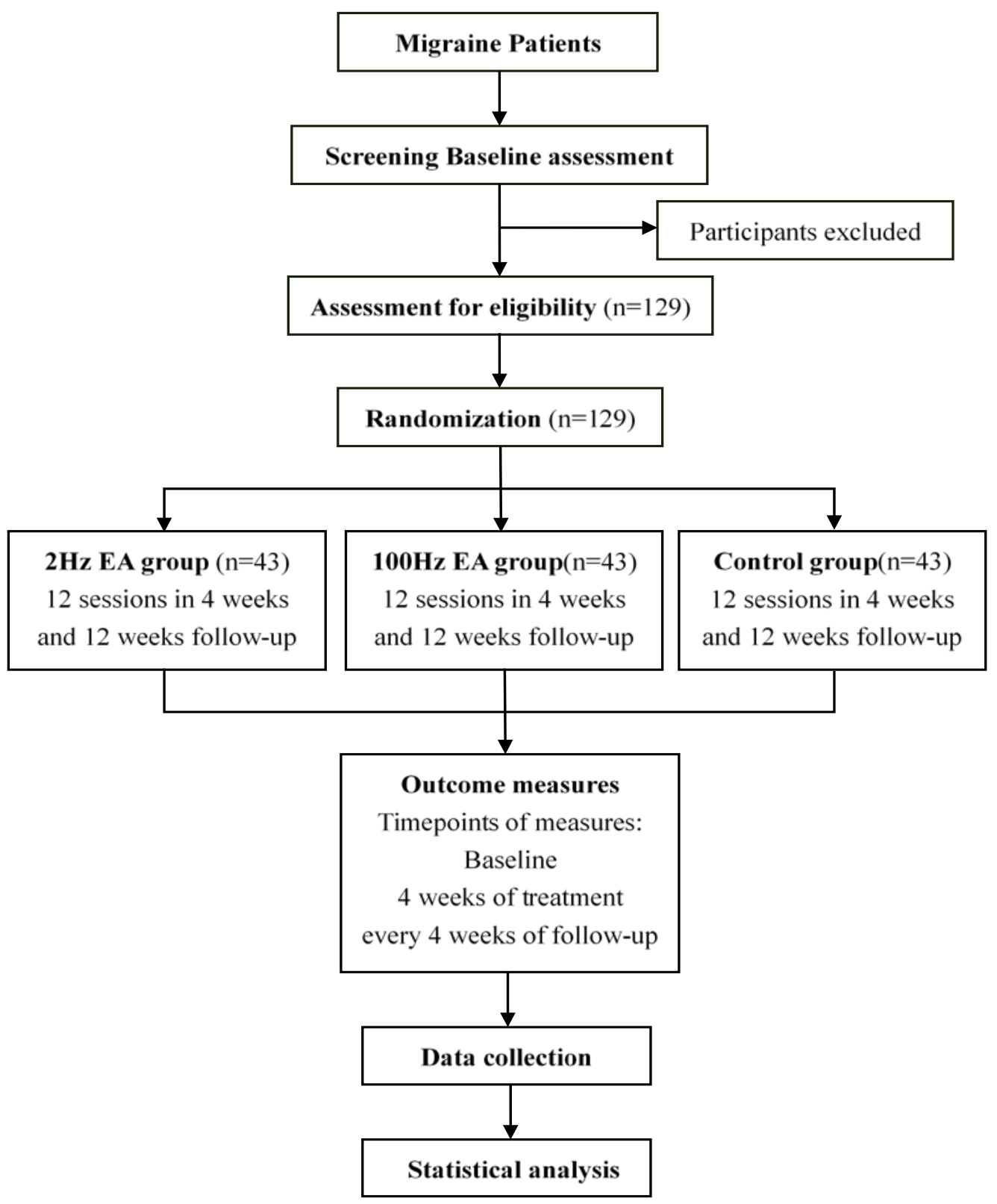

Figure 1. Flow diagram of study design

\section{Participant recruitment}

Patients will be recruited in the Third Affiliated Hospital of Zhejiang Chinese Medicine University in Zhejiang Province, China. We will propagate by posters in hospitals and the internet. If patients are interested in the study, they will be examined for eligibility by a researcher and informed they can withdraw from the study at any time. And prospective participants will obtain written informed consent and administer the baseline observation followed by random allocation. 


\section{Inclusion criteria}

All collected migraine patients will diagnosed with criteria ofthe International Headache Classification (3rd edition,ICHD- III ), which set by International Headache Society (IHS)[23].Participants must also match the following criteria: (1) those aged 18-65 years with more than 1 year of migraine history; (2) suffering from 2-8 times headache attacks per month and less than 15 headache days per month on average during the previous 3 months. (3) having the ability to complete the headache diary; (4) written informed consent was provided by themselves or guardians.

\section{Exclusion criteria}

Participants meeting any of the following criteria will be included: (1) headache resulted from craniocerebral disease such as cerebral vascular diseases, intracranial aneurysms, space-occupying lesions and other intracranial organic diseases; (2) headache caused by cluster headache, hemiplegic or basilar migraine; (3) have taken prophylactic medication (eg. beta-adrenoceptor blockers, calcium antagonists, antiepileptic drugs, antidepressant drugs, 5-HT receptor blockers) or received acupuncture treatment in the previous 1 month; (4) having a mental illness or a serious cognitive impairment; (5) suffering from any serious disease of cardiovascular, cerebrovascular, hepatic, renal, hematopoietic systems; (6) those who are pregnant or lactating, or planning to become pregnant; (7) those had participated in the other clinical trials at the same time.

\section{Ethical considerations}

The protocol of this study was approved by the Medical Ethics Committee of the Third Affiliated Hospital of Zhejiang Chinese University(permission number: ZSLL-KY-2017-036). All participants or guardians will be giving full explanation for the purpose, nature, and potential risks of the study and sign the informed consent before the experiment.

\section{Randomization and blinding}

Randomization will be conducted by independent research staff who will have no contact with the patients. The random number generated on the RAMDOM (https://www.random.org). The randomized codes will be kept in sealed andopaque envelopes and randomly allocated when researcher informed of a participant's enrollment. All participants and study researchers including evaluator and data analysts will be blinded to group assignments. However, it is impossible to blind the acupuncturist, which will not take part in any evaluation process.

After the data reviewed, the first unblinding will be carried out, and the second unblinding will be conducted after the data analysis. In case of emergency or the subject needs to be rescued, the researcher will disassemble it according to the procedures in the protocol. Once disassembled, the numbered case will be discontinued, and the researcher should record the reason for suspension in the Case Report Form.

\section{Interventions}

The study is a randomized clinical trial carried out in the outpatients of the Third Affiliated Hospital of Zhejiang Chinese Medicine University. Participants will be randomized to $2 \mathrm{~Hz}$ EA group, $100 \mathrm{~Hz}$ EA group and control group and will receive 12 sessions of treatment over 4 weeks. Participants in the two EA group will receive $2 \mathrm{~Hz}$ or $100 \mathrm{H}$ EA stimulation respectively, 
while those in the control group will receive superficial acupuncture in non-acupoints withsham stimulation of EA. The acupuncturist will be allowed to participate in the trial at the completion of training.The locationand specific operation of EA groups and control group areshown in Table 1.

Table 1 Details of the EA group and control group

\begin{tabular}{|c|c|c|}
\hline Group & Acupoint & Manipulation \\
\hline \multirow[t]{7}{*}{$\begin{array}{c}\text { EA Group } \\
(2 \mathrm{~Hz} \text { and100 Hz) }\end{array}$} & (i)Fengchi (GB20) & $\begin{array}{l}\text { (i) is punctured obliquely } 15-30 \mathrm{~mm} \\
\text { toward to apex nasi }\end{array}$ \\
\hline & (ii)Gongxue (extra-point) & $\begin{array}{l}\text { (ii) is punctured obliquely } 15-30 \mathrm{~mm} \\
\text { toward to apex nasi }\end{array}$ \\
\hline & (iii)Shuaigu (GB8) & $\begin{array}{l}\text { (iii) is punctured horizontally } 10-20 \mathrm{~mm} \\
\text { backward }\end{array}$ \\
\hline & (iv) Taiyang (EX-HN5) & $\begin{array}{l}\text { (iv) is punctured obliquely } 7.5-15 \mathrm{~mm} \\
\text { backward }\end{array}$ \\
\hline & (v)Sizhukong (SJ23) & $\begin{array}{l}\text { (v) is punctured obliquely } 10-20 \mathrm{~mm} \\
\text { backward }\end{array}$ \\
\hline & (vi) Waiguan (SJ5) & $\begin{array}{l}\text { (vi) is punctured perpendicularly } 10-20 \\
\mathrm{~mm}\end{array}$ \\
\hline & (vii) Yanglingquan(GB34) & $\begin{array}{l}\text { (vii) is punctured perpendicularly 25- } \\
40 \mathrm{~mm}\end{array}$ \\
\hline \multirow[t]{9}{*}{ Control Group } & $\begin{array}{l}\text { (i) Fengchi (GB20) outward } \\
1 \mathrm{~cm} \text { by side }\end{array}$ & $\begin{array}{l}\text { (i)is punctured obliquely } 7.5-15 \mathrm{~mm} \\
\text { toward to apex nasi }\end{array}$ \\
\hline & $\begin{array}{l}\text { (ii) Gongxue (extra-point) } \\
\text { outward } 1 \mathrm{~cm} \text { by side }\end{array}$ & $\begin{array}{l}\text { (ii)is punctured obliquely } 7.5-15 \mathrm{~mm} \\
\text { toward to apex nasi }\end{array}$ \\
\hline & (iii)Shuaigu (GB8) backward & (iii) is punctured horizontally $5-7.5 \mathrm{~mm}$ \\
\hline & $1 \mathrm{~cm}$ by side & backward \\
\hline & $\begin{array}{l}\text { (iv) Taiyang (EX-HN5) } \\
\text { backward } 1 \mathrm{~cm} \text { by side }\end{array}$ & $\begin{array}{l}\text { (iv) is punctured obliquely } 5-7.5 \mathrm{~mm} \\
\text { backward }\end{array}$ \\
\hline & $\begin{array}{l}\text { (v) Sizhukong (SJ23) } \\
\text { backward } 1 \mathrm{~cm} \text { by side }\end{array}$ & $\begin{array}{l}\text { (v) is punctured obliquely } 5-7.5 \mathrm{~mm} \\
\text { backward }\end{array}$ \\
\hline & $\begin{array}{l}\text { (vi) Waiguan (SJ5) outward } \\
\text { (radial side) } 1 \mathrm{~cm} \text { by side }\end{array}$ & $\begin{array}{l}\text { (vi)is punctured perpendicularly 5-10 } \\
\mathrm{mm}\end{array}$ \\
\hline & (vii) Yanglingquan (GB34) & (vii)is punctured perpendicularly $7.5-15$ \\
\hline & outward $1 \mathrm{~cm}$ by side & $\mathrm{mm}$ \\
\hline
\end{tabular}

\section{$2 H z$ EA group}

The acupoints prescription (Table 1 ) is designed by an experienced acupuncturist who spend more than 30 years in practicing electroacupuncture analgesia. The local skin of all acupoints should been disinfected when patients arein a comfortablesitting position. Sterile needles (0.25 $\mathrm{mm}$ in diameter, $40 \mathrm{~mm}$ in length, Suzhou Medical Appliance in Suzhou, Jiangsu Province, China) will be inserted into each acupoint and the depths will be adjusted by acupuncturist until the arrival of deqi sensation. Then Fengchi(GB20) and Gongxue(extra-point) in homolateral will be connected to the output terminals of the HANS Acupuncture point Nerve Stimulators (HANS-200A, Nanjing Jisheng Medical Technology Co., 
Ltd., Nanjing, Jiangsu Province, China) and stimulated with low-frequency $(2 \mathrm{~Hz})$ EA and appropriate endurable intensity. The EA treatment will be given once every other day and be retained for 30 minutes in each session.

\section{$100 \mathrm{~Hz}$ EA group}

The EA frequency is set to $100 \mathrm{~Hz}$, but beyond that, acupoint selection and specific operation both are the same as the $2 \mathrm{~Hz}$ EA group.

\section{Control group}

Appropriate acupuncture points about $1 \mathrm{~cm}$ nearbyare selected and superficial needlingare operated for the control group.That means meridians and other acupoints should be avoided. We do notrequire the arrival of Qi. Meanwhile, sham EA (the output circuit of apparatus will be cut off) will be operated during the trial period as same as real EA besides electrical stimulation.

\section{Outcome measures}

The following outcomes will be measured by independentassessors blinded to the allocation. The patients will be carefully examined at baseline and reexamined after four weeks of treatment. Follow-up will be performed 4, 8 and 12 weeks after completion of treatment. Detailed time points of outcome measures are provided in Table 2.

Table 2 Timetable of treatment and outcome collection

\begin{tabular}{|c|c|c|c|c|c|}
\hline \multirow{2}{*}{$\begin{array}{c}\text { Period } \\
\text { Visit }\end{array}$} & \multirow{2}{*}{$\frac{\text { Baseline }}{1}$} & \multirow{2}{*}{$\begin{array}{c}\begin{array}{c}\text { Treatment } \\
\text { phase }\end{array} \\
2\end{array}$} & \multicolumn{3}{|c|}{ Follow-up phase } \\
\hline & & & 3 & 4 & 5 \\
\hline Week & $1-4$ & $5-8$ & $9-12$ & $13-16$ & $17-20$ \\
\hline Informed consent & $x$ & & & & \\
\hline Demographic characteristics & $x$ & & & & \\
\hline Medical history & $\times$ & & & & \\
\hline Inclusion/exclusion criteria & $x$ & & & & \\
\hline $\begin{array}{c}\text { Acupuncture } \\
\text { expectancy questionnaire }\end{array}$ & $\times$ & & & & \\
\hline Random allocation & $x$ & & & & \\
\hline EA & & \# & & & \\
\hline Frequency of migraine attacks & $x$ & $x$ & $x$ & $x$ & $\times$ \\
\hline Number of days with migraine & $\times$ & $x$ & $\times$ & $x$ & $x$ \\
\hline Frequency of taking ibuprofen & $x$ & $x$ & $x$ & $x$ & $\times$ \\
\hline VAS & $x$ & $x$ & $x$ & $\times$ & $x$ \\
\hline SAS & $x$ & $x$ & $x$ & $x$ & $x$ \\
\hline SDS & $x$ & $x$ & $x$ & $\times$ & $x$ \\
\hline MSQ & $x$ & $x$ & $x$ & $\times$ & $x$ \\
\hline Safety assessment & & & & & $x$ \\
\hline Compliance evaluation & & & & & $x$ \\
\hline
\end{tabular}

$\times$, All groups; \#, EA groups. 


\section{Baseline measures}

Baseline measures will be conducted before randomization, including gender, age, height, weight, duration of disease, family history. The measures include patients whether the patient had received acupuncturetreatmentand expectation of acupuncturetreatment for patients.

\section{Primary outcome measure}

The primary outcome measure is frequency of migraine attacks, which will be recorded at baseline, treatment and three follow-ups.

\section{Secondary outcome measures}

The secondary outcomes are evaluated with number of days with migraine,frequency of taking ibuprofen,VAS, SAS, SDS and MSQ, which will be recorded the same five periods.

\section{Incidence of adverse events}

Any adverse event(AE) and serious adverse event (SAE), and how they are dealt with, will be recorded during the four-week treatment and three consecutive four-week follow-up. AEs include bleeding, hematoma, fainting, severe pain, and local infection. Any SAE can lead to driving the disease process. If participants suffer AEs or SAEs, all details such as the date of occurrence, time, degree, measurement related to the treatment, course, outcome and causal relationship with the treatment will be documented. Moreover, if SAEs occur in participants, physicians should immediately provide the appropriate care to ensure their safety. Additionally, SAEs will be reported to principal investigatorimmediately and also to the clinical center and the medical ethics committee in 24 Hours.

\section{Quality control}

The protocol has been perfected by experienced acupuncturists and statisticians before the trial.In addition, all researchers are required to take part in a series of training sessions including research protocol, standard operating procedures and acupuncture practical lessons, etc. There will bearranged a monitor to supervise the operators and discuss the progress every 3 months during the trial.Data inputs and storage are performed in Research Manager (ResMan, http://www.medresman.org). And we will input the data with double entry for ensuring the accuracy.Principal investigator and the statistician will have access to all data in the research. If serious safety problems occur in the trial, such as serious adverse events or serious complications, the trial will be terminated. Principal investigator will make the final decision to the termination.

\section{Sample size calculation}

Sample size of this study is estimated by the method of multiple samples mean comparison. For this trial, it has been determined that $\alpha=0.05,1-\beta=0.90$ and then we looked up table that $\Phi=2.52$. According to a reference[24], we anticipated that the improvement mean of migraine frequency attack in $2 \mathrm{~Hz}$ and $100 \mathrm{~Hz}$ EA groups are 3.26 , whereas, in the non-acupoint group is 1.48. The standard deviation of two EA group and non-acupoint group are 2.28 and 2.57 respectively. There are three groups in total that $\mathrm{k}=3$. The sample size will be calculated by the equation as below.

$$
n=\psi^{2}\left(\Sigma S_{i}^{2} / k\right) /\left[\Sigma\left(\bar{X}_{i}-\bar{X}\right)^{2} /(k-1)\right]
$$


Thus, at least 34 participants are required for each group. To compensate for a prevalence of withdrawal of 20\%, we plan to recruit 129 participants in the three groups, with 43 patients for each group.

\section{Statistical analysis}

All data analysis will performed by the SPSS statistical program (version 19.0, Chicago, Illinois, United States). And we will express the results of statistical analysis with the principle of intention-to-treat (ITT). Normally distributed variables will be expressed as mean $\pm S D$. Analysis of variance (ANOVA) for repeated measures will be used for numerical variables and the Chi-square test $\left(X^{2}\right.$ test) for categorical variables. The analysis results will be considered to indicate statistical significance when $P<0.05$.

\section{Discussion}

Acupuncture, as the vital part of traditional Chinese medicine, has been becoming more and more popular all over the world. Moreover, acupuncture has been registered and accredited in many countries. EA is a novel type of acupuncture and becomes more acceptable than traditional acupuncture due to stronger analgesic potency and parameter-adjustable feature.

Previous systematic reviews and clinical trials had indicated that acupuncture and EA exerted favorable analgesic effect on migraine [19-22]. Previous studies showed that the underlying mechanism between low-frequency and high-frequency EA had distinct. Low-frequency EA release enkephalin and endorphin which can last for a long time whereas high-frequency EA release dynorphin rapidly which get the analgesia effect immediately [16]. According to professor Han's achievements in scientific research, $2 \mathrm{~Hz}$ EA had been recommended to treat neuropathic pain[16].Giventhe lack of trials of EA with different frequencies for migraine, the frequency preference of EA for treating migraine remains uncertain. Therefore, we designed a parallel-design, patient-assessor blind, RCT to explore the dominant frequency of EA on migraine. Studies had shown that negative emotion has a close relationship with pain diseases and changes on pain-related affection had been improved by acupuncture [25-28]. In this trial, psychological and emotional condition of participants will be assessed by using SAS and SDS scale.Migraine Specific Quality of Life questionnaire (MSQ) is the specific evaluation method more widely used for quality of life in migraine patients. As paininfluences the life quality of the patients, we designed MSQ for evaluation of quality of life[6, 11, 12].

Non-acupoint group was operated by superficial needling for non-acupoint and sham EA in this trial. Although all steps of sham EA manipulation are equally to real EA besides electrical stimulation which will cut offthe output circuit, blinding of real and sham EA treatment seem difficult and even impossible because of the patients may finally discovering their treatment for placebo-control. For clinical trials, blinding is difficult for acupuncture or EA, so real randomized trials seem impossible.To improve compliance of participants, they are allowed to take ibuprofen in necessary and will take 12 sessions treatment for free. Moreover, we formulate the project to improve compliance by establishing good communication relationships via text messages and telephone calls during the 20 weeks of baseline, treatment and follow-up period.

Briefly, the aim of this trial is to confirm the analgesic effect of low- and high-frequency EA 
and to further explore the dominant frequency of EA for migraine. This study may also confirm the therapeutic difference of low- and high-frequency EA on psychological and emotional changes due to headache attacks.

\section{Trial status}

Participant enrollment started on 1 August 2018. The completion are expected to be finished by the end of 2021 (extend 1 year due to COVID-19).This protocol has been revised three times for the version number 3.0, and date of the latest revision is 21 June 2020.

\section{Abbreviations}

TCM: Traditional Chinese Medicine; EA: Electroacupuncture; Hz: Hertz (cycles per second); WHO: World Health Organization; CNY: China Yuan; USD: United States dollar; GAD: generalized anxiety disorder; ICHD-3: International Classification of Headache Disorders, 3rd Edition; VAS: Visual Analogue Scale; SAS: Self-Rating Anxiety Scale; SDS: Self-Rating Depression Scale; MSQ: Migraine Specific Quality of Life Questionnaire; SPSS: Statistical Product and Service Solutions; AE: adverse event; SAE: serious adverse event.

\section{Competing interests}

Non-financial competing interests was declared by all the authors.

\section{Authors' contributions}

$\mathrm{Na}$ Nie and Le Chen drafted the protocol. Yi Liang and Jianqiao Fang revised the manuscript and contributed to the conception and design of the trial. Tong Li, Chuanlong Zhou and Bangwei Li participated in data collection. Jie Zhou and Qin Chen are in charge of recruitment of patients. Conghua Ji and Qiushuang Li are planning the analysis of the data. All the authors read and approved the final manuscript.

\section{Ethics approval and consent to participate}

The study is approved by Medical Ethics Committee of the Third Affiliated Hospital of Zhejiang Chinese University (Approval No. ZSLL-KY-2017-036), and registered in Chinese Clinical Trial Registry (Registration No. ChiCTR-1800017259). All patients provide written informed consent form to participate in this study.Once the important protocol needs to be modified, all members of the research team will participate it and submitthe final modification to MedicalEthics Committee for review.

\section{Funding}

This study is supported by the Foundation of Health Commission of Zhejiang Province (No.2018ZA060, 2020ZB125). Sponsor has no role in design of the study; collection, analysis, interpretation of data and writing of the report; and the decision to submit the report for publication. Also, they do not have ultimate authority over any process.

\section{Acknowledgements}

We are particularly grateful to anyone who provided helpful advice on the design of the trial. Meanwhile, we appreciate the help from the patients participating in the trial, including who contributed towards the trial who does not meet the criteria for authorship.

\section{Author details}

1 Department of Acupuncture, The Third Affiliated Hospital of Zhejiang Chinese Medical 
University, No. 219 Moganshan Road, XiHu District, Hangzhou, Zhejiang Province 310005, China. 2 The Third Clinical Medical College of Zhejiang Chinese Medical University, No. 548 Binwen Road, Binjiang District, Hangzhou, Zhejiang Province 310053, China.

3 The Clinical Research Institute of Zhejiang Provincial Hospital of TCM, No.54 Youdian Road, XihuDistrict, Hangzhou, Zhejiang Province 310006, China.

\section{References}

1. Lipton RB, Bigal ME, Diamond M, Freitag F, Reed ML, Stewart WF, Group AA: Migraine prevalence, disease burden, and the need for preventive therapy. Neurology 2007, 68(5):343-349.

2. Lipton RB, Stewart WF, Diamond S, Diamond ML, Reed M: Prevalence and Burden of Migraine in the United States: Data From the American Migraine Study II. Headache 2001, 41(7):646-657.

3. Goadsby $\mathrm{P}, \mathrm{T}$.: Recent advances in the diagnosis and management of migraine $B M J$ 2006, 332:25-29.

4. Menken M, Munsat TL, Toole JF: The Global Burden of Disease Study: Implications for Neurology. JAMA Neurology 2016, 57:418-420.

5. Sayers J: The world health report 2001 - Mental health: new understanding, new hope. Bulletin of the World Health Organization 2001, 79(11):1085.

6. Yu SY, Liu RZ, Zhao G, Yang XS, Qiao XY, Feng JC, Fang YN, Cao XT, He MW, Steiner T: The Prevalence and Burden of Primary Headaches in China: A Population-Based Door-to-Door Survey. Headache: The Journal of Head and Face Pain 2012, 52(4):582-591.

7. Collaborators. GH: Global, regional, and national burden of migraine and tension-type headache, 1990-2016: a systematic analysis for the Global Burden of Disease Study 2016. The Lancet Neurology 2018, 17(11):954-976.

8. Lipton RB, Diamond S, Reed M, Diamond ML, Stewart WF: Migraine Diagnosis and Treatment: Results From the American Migraine Study II. Headache 2001, 41:638-645.

9. Hazard E, Munakata J, Bigal ME, Lipton RB, Rupnow MFT: The Burden of Migraine in the United States: Current and Emerging Perspectives on Disease Management and Economic Analysis. Value In Health 2009, 12(1):55-64.

10. Steiner TJ, Stovner LJ, VOS T: GBD 2015: migraine is the third cause of disability in under 50s. The journal of headache and pain 2016, 17(1).

11. Smitherman TA, Burch R, Sheikh H, Loder E: The prevalence, impact, and treatment of migraine and severe headaches in the United States: a review of statistics from national surveillance studies. Headache 2013, 53(3):427-436.

12. Leonardi M, Steiner TJ, Scher AT, Lipton RB: The global burden of migraine: measuring disability in headache disorders with WHO's Classification of Functioning, Disability and Health (ICF). The journal of headache and pain 2005, 6(6):429-440.

13. Hepp Z, Bloudek LM, Varon SF: Systematic review of migraine prophylaxis adherence and persistence. Journal of managed care pharmacy: JMCP 2014, 20(1):22-33.

14. Liang Y, Qiu YJ, Du JY, Liu J, Fang JF, Zhu J, Fang JQ: Inhibition of spinal microglia and astrocytes contributes to the anti-allodynic effect of electroacupuncture in neuropathic pain induced by spinal nerve ligation. Acupuncture in medicine : journal 
of the British Medical Acupuncture Society 2016, 34(1):40-47.

15. Liang Y, Fang JQ, Du JY, Fang JF: Effect of Electroacupuncture on Activation of p38MAPK in Spinal Dorsal Horn in Rats with Complete Freund's Adjuvant-Induced Inflammatory Pain. Evidence-based complementary and alternative medicine : eCAM 2012, 2012:568273.

16. Han JS: New Evidence to Substantiate the Frequency Specificity of Acupuncture-induced Analgesia. Acupuncture Research 2001, 26(3):224-227.

17. Xiang XE, Du JY, Fang JF, Liu YJ, XU ZT, Wang SS, Sun HJ, Fang JQ: Selection of optimal frequency on electroacupuncture analgesia and research of the mechanism of TRPV1 activation in the spinal cord dorsal horn at the early phase of different types of pathological pain. China Journal of Traditional Chinese Medicine and Pharmacy 2018, 33(5):1707-1712.

18. Sun RQ, Wang HC, Wang $\mathrm{Y}$, Luo F, Han JS: Effect of electroacupuncture with different frequencies on Neuropathic pain in a rat model. Chinese Journal of Applied Physiology 2002, 18(2):128-131.

19. Li YX, Xiao XL, Zhong DL, Luo LJ, Yang H, Zhou J, He MX, Shi LH, Li J, Zheng $H$ et al: Effectiveness and Safety of Acupuncture for Migraine: An Overview of Systematic Reviews. Pain research \& management 2020, 2020:3825617-3825617.

20. Zhang XT, Li XY, Zhao C, Hu YY, Lin YY, Chen HQ, Shi ZF, Zhang XY, Shang HC, Tian GH: An Overview of Systematic Reviews of Randomized Controlled Trials on Acupuncture Treating Migraine. Pain research \& management 2019, 2019:5930627.

21. Li X, Dai Q, Shi Z, Chen H, Hu Y, Wang X, Zhang X, Tian G: Clinical Efficacy and Safety of Electroacupuncture in Migraine Treatment: A Systematic Review and Network Meta-Analysis. Am J Chin Med 2019, 47(8):1755-1780.

22. Zhao L, Chen J, Li Y, Sun X, Chang X, Zheng H, Gong B, Huang Y, Yang M, Wu X et al: The Long-term Effect of Acupuncture for Migraine Prophylaxis: A Randomized Clinical Trial. JAMA Intern Med 2017, 177(4):508-515.

23. IHS: The International Classification of Headache Disorders, 3rd edition. Cephalalgia: an international journal of headache 2018, 38(1):1-211.

24. Chen J: Evaluating the prophylaxis and long-term effectiveness of meridian-based acupuncture for migraine: A randomized controlled trial. Chengdu: Chengdu University of Traditional Chinese Medicine; 2014.

25. Zhang RX, Lao LX, Ren K, Berman BM: Mechanisms of acupuncture-electroacupuncture on persistent pain. Anesthesiology 2014, 120(2):482-503.

26. Chifumi I, Taiga F, Masao S, Shingo K, Keiji S, Tomoko S: Acupuncture Alleviated the Nonmotor Symptoms of Parkinson's Disease including Pain, Depression, and Autonomic Symptoms. Case reports in neurological medicine 2014, 2014:953109.

27. Baeumler PI, Fleckenstein J, Takayama S, Simang M, Seki T, Irnich D: Effects of acupuncture on sensory perception: a systematic review and meta-analysis. PloS one 2014, 9(12):e113731.

28. Xiang $\mathrm{XH}$, Chen $\mathrm{YM}$, Zhang JM, Tian JH, Han JS, Cui CL: Low- and high-frequency transcutaneous electrical acupoint stimulation induces different effects on cerebral mu-opioid receptor availability in rhesus monkeys. Journal of neuroscience research 
2014, 92(5):555-563. 